\title{
SOME ARITHMETIC FUNCTIONS OF FACTORIALS IN LUCAS SEQUENCES
}

\author{
Eric F. Bravo AND Jhon J. Bravo \\ Universidad del Cauca, Colombia
}

\begin{abstract}
We prove that if $\left\{u_{n}\right\}_{n \geq 0}$ is a nondegenerate Lucas sequence, then there are only finitely many effectively computable positive integers $n$ such that $\left|u_{n}\right|=f(m !)$, where $f$ is either the sum-of-divisors function, or the sum-of-proper-divisors function, or the Euler phi function. We also give a theorem that holds for a more general class of integer sequences and illustrate our results through a few specific examples. This paper is motivated by a previous work of Iannucci and Luca who addressed the above problem with Catalan numbers and the sum-of-proper-divisors function.
\end{abstract}

\section{INTRODUCTION}

Let $\left\{u_{n}\right\}_{n \geq 0}$ be a Lucas sequence, i.e., a sequence given by $u_{0}=0, u_{1}=1$ and the binary linear recurrence

$$
u_{n}=a u_{n-1}+b u_{n-2} \text { for all } n \geq 2,
$$

where $a$ and $b$ are non-zero coprime integers such that the discriminant $\Delta:=$ $a^{2}+4 b \neq 0$. Denote by $\alpha$ and $\beta$ the roots of the characteristic polynomial $f(X)=X^{2}-a X-b$ and suppose without loss of generality that $|\beta| \leq|\alpha|$. Note that $\alpha$ and $\beta$ are distinct and non-zero. It is well known that the Binettype formula

$$
u_{n}=\frac{\alpha^{n}-\beta^{n}}{\alpha-\beta} \quad \text { holds for all } n \geq 0 .
$$

A Lucas sequence $\left\{u_{n}\right\}_{n \geq 0}$ is called degenerate if the quotient $\alpha / \beta$ of the roots of $f$ is a root of unity and nondegenerate otherwise. The divisibility

2020 Mathematics Subject Classification. 11A25, 11B39.

Key words and phrases. Lucas sequence, arithmetic function, Diophantine equation.

J. J. B. was supported in part by Project VRI ID 5385 (Universidad del Cauca). 
properties of the integers $u_{n}$ have been studied by Euler, Lagrange, Gauss, Dirichlet and others (see [4, Chapter XVII]).

Some Lucas sequences are so important that they have special names. For example, taking $(a, b)=(1,1)$, the sequence $\left\{u_{n}\right\}_{n \geq 0}$ becomes the classical Fibonacci sequence $\left\{F_{n}\right\}_{n \geq 0}$ for which $\alpha=(1+\sqrt{5}) / 2$ and $\beta=(1-\sqrt{5}) / 2$. If $(a, b)=(2,1)$, then the Pell sequence $\left\{P_{n}\right\}_{n \geq 0}$ appears for which $\alpha=1+\sqrt{2}$ and $\beta=1-\sqrt{2}$.

Let $m$ be a positive integer. Some of the most familiar arithmetic functions in number theory are:

- the divisor-sum function $\sigma(m)$, which sums the divisors of $m$. Its first values appear as A000203 in the Sloane's On-Line Encyclopedia of Integer Sequences (OEIS) [21];

- $s(m)$ denoting the sum of the aliquot parts of $m$, i.e., the sum of the proper divisors of $m$, or equivalently, $s(m)=\sigma(m)-m$. The first few values of $s(m)$ are therefore $0,1,1,3,1,6,1,7,4, \ldots$ (OEIS A001065);

- the Euler phi function $\phi(m)$, which represents the number of positive integers $\leq m$ and coprime with $m$ (OEIS A000010).

The study of arithmetic functions of a linearly recurrent sequence has been a popular area of research. For example, in [10] it was shown that 1, 2, and 3 are the only Fibonacci numbers whose Euler function is also a Fibonacci number, while in [3] it was proved that if $\left\{u_{n}\right\}_{n \geq 0}$ is a Lucas sequence with $b \in\{ \pm 1\}$, then there are only finitely many effectively computable $n$ such that $\phi\left(\left|u_{n}\right|\right)$ is a power of 2 , extending the previous works $[8,12]$ which dealt with the above problem for the particular Lucas sequences of the Fibonacci and Pell numbers. In [9] it was shown that the largest Fibonacci number whose Euler function is a repdigit (i.e., numbers with only one distinct digit in its decimal expansion) is $F_{11}$ for which $\phi\left(F_{11}\right)=88$, while in [11] it was proved that there are no repdigits with two or more digits which are multiply perfect, namely numbers who divide the sum of their divisors.

In 2007, Iannucci and Luca ([5]) looked for factorials whose sum of aliquot parts is a Catalan number. In fact, they proved that the only solution in positive integers $(n, m)$ for the Diophantine equation $C_{n}=s(m !)$ is the trivial solution $(1,2)$. We recall that a Catalan number is a number of the form $C_{n}=\frac{1}{n+1}\left(\begin{array}{c}2 n \\ n\end{array}\right)$ for $n \geq 0$ (OEIS A000108).

In this paper, we consider an equation similar to that of Iannucci and Luca ([5]), but with members of Lucas sequences instead of Catalan numbers and including other arithmetic functions. Specifically, we are interested in studying the Diophantine equation

$$
\left|u_{n}\right|=f(m !)
$$

in positive integers $(n, m)$, where $f$ is either the sum-of-divisors function, or the sum-of-proper-divisors function, or the Euler phi function. 
We begin by mentioning that if $\left\{u_{n}\right\}_{n \geq 0}$ is a degenerate Lucas sequence, then it is known $([16$, pp. 5-6]) that

$$
(a, b) \in\{( \pm 2,-1),( \pm 1,-1),(0, \pm 1),( \pm 1,0)\},
$$

and in these cases $\left\{u_{n}\right\}_{n \geq 0}$ is either periodic with values in $\{0,-1,1\}$, or equal to $\{n\}_{n \geq 0}$, or equal to $\left\{(-1)^{n-1} n\right\}_{n \geq 0}$, so the problem (1.1) becomes trivial. Thus, for the rest of the paper we assume that $\left\{u_{n}\right\}_{n \geq 0}$ is a nondegenerate Lucas sequence and so $|\alpha|>1$.

We prove the following result.

THEOREM 1.1. Let $\left\{u_{n}\right\}_{n \geq 0}$ be a nondegenerate Lucas sequence and consider the Diophantine equation (1.1) where $f \in\{\sigma, s, \phi\}$. Then $\max \{n, m\}$ is bounded by an effectively computable number depending only on a and $b$.

As we shall see later, from the proof of Theorem 1.1, we can extract some expressions which allow us to get upper bounds on the solutions of the Diophantine equation (1.1). We will illustrate this method in Section 4 by solving the equation (1.1) in some particular cases of the sequence $\left\{u_{n}\right\}_{n>0}$.

At the end of the paper we work in a more general setting of integer sequences. We exploit some ideas developed in this work to prove, under some technical conditions on the sequence $\left\{u_{n}\right\}_{n \geq 0}$, the finiteness of the number of solutions of equation (1.1).

\section{Auxiliary Results}

In this section, we present some auxiliary results that are needed in the proofs of the main theorems. We begin by collecting some facts that we need about Lucas sequences. The first lemma gives us a good lower bound for $\left|u_{n}\right|$ in terms of $|\alpha|$ and can be deduced from [20, Lemma 3] (see also [19, Lemmas 1 and 2]).

Lemma 2.1. There exists a positive constant $c$ such that

$$
\left|u_{n}\right| \geq|\alpha|^{n-c \log (n+1)} \quad \text { for all } n \geq 1 .
$$

The proof of Lemma 2.1 depends upon an estimate for a linear form in the logarithms of two algebraic numbers due to Baker, [1].

Let $p$ be a prime number. Recall that if $p \nmid b$, then the integer

$$
\tau(p):=\min \left\{k \geq 1: p \mid u_{k}\right\}
$$

exists and is called the rank of apparition of $p$ in $\left\{u_{n}\right\}_{n \geq 0}$. This has the remarkable property that $p \mid u_{n}$ if and only if $\tau(p) \mid n$ whenever $n>1$ (see, e.g., [15]).

For a non-zero integer $k$, the $p$-adic valuation of $k$, commonly denoted by $v_{p}(k)$, is the highest exponent $r$ such that $p^{r}$ divides $k$. The $p$-adic valuation of Lucas sequences has been studied before by many authors. We first cite the results presented by Lucas in 1878 of his massive journal paper [13], in which 
we find implicit interesting divisibility properties known nowadays in compact form. The $p$-adic order of the Fibonacci numbers was completed characterized by Lengyel in [7]. In 2016, Sanna ([18, Theorem 1.5]) gave simple formulas for the $p$-adic order $v_{p}\left(u_{n}\right)$, in terms of $v_{p}(n)$ and the rank of apparition of $p$ in $\left\{u_{n}\right\}_{n \geq 0}$, where $\left\{u_{n}\right\}_{n \geq 0}$ is a nondegenerate Lucas sequence. It was proved in $[18]$ that

THEOREM 2.2. If $p \nmid b$, then

$$
v_{p}\left(u_{n}\right)= \begin{cases}v_{p}(n)+v_{p}\left(u_{p}\right)-1, & \text { if } p|\Delta, p| n, \\ 0, & \text { if } p \mid \Delta, p \nmid n, \\ v_{p}(n)+v_{p}\left(u_{p \tau(p)}\right)-1, & \text { if } p \nmid \Delta, \tau(p)|n, p| n, \\ v_{p}\left(u_{\tau(p)}\right), & \text { if } p \nmid \Delta, \tau(p) \mid n, p \nmid n, \\ 0, & \text { if } p \nmid \Delta, \tau(p) \nmid n,\end{cases}
$$

for each positive integer $n$.

The following property of the $p$-adic valuation of $\left\{u_{n}\right\}_{n \geq 0}$ was also derived by Sanna ([18, Lemma 3.2]) and we will be used later to find an upper bound for $v_{2}\left(u_{n}\right)$.

LEMMA 2.3. If $p \nmid b$, then

$$
v_{p}\left(u_{p \tau(p)}\right) \geq v_{p}\left(u_{\tau(p)}\right)+1
$$

with equality if either $p \geq 5$, or $p=3$ and $3 \nmid \Delta$.

The next lemma is a consequence of Legendre's formula whose proof can be found in [14]. For a real number $x$, we denote by $\lfloor x\rfloor$ the largest integer less than or equal to $x$.

LEMmA 2.4. For any integer $r \geq 1$ and prime $p$, we have

$$
\frac{r}{p-1}-\left\lfloor\frac{\log r}{\log p}\right\rfloor-1 \leq v_{p}(r !) \leq \frac{r-1}{p-1} .
$$

We finish this section with the following result on the prime counting function $\pi(x)$, which gives the number of primes $p \leq x$. This result was found by Rosser and Schoenfeld ([17, Corollary 3]) and will be used in the following section to find a lower bound for the 2-adic valuation of $f(m !)$.

LEMma 2.5. For $x \geq 20.5$, we have that

$$
\pi(2 x)-\pi(x)>3 x /(5 \log x) .
$$

\section{Proof of Theorem 1.1}

Suppose first that $(n, m)$ is a positive integer solution of equation (1.1). If $m \leq 50$, then $n$ has finitely many possibilities by Lemma 2.1 , and thus there is nothing to prove (In fact, the number of times that $u_{n}$ assumes a given integer 
value is $\leq 4$ by the main result in [6] (see also [2] for an improvement)). So, we will assume that $m>50$. The main idea of the proof is to compare the 2-adic valuation on both sides of (1.1).

Lemma 3.1. If $m>50$, then

$$
v_{2}(f(m !))>\frac{3 m}{10 \log m} .
$$

Proof. Since $m$ ! is divided exactly once by all primes $p$ such that $m / 2<$ $p \leq m$, it follows that

$$
\begin{aligned}
v_{2}(\sigma(m !)) & \geq \sum_{m / 2<p \leq m} v_{2}(p+1) \\
& \geq \pi(m)-\pi(m / 2) \\
& >\frac{3 m}{10 \log m} \text { for all } m>50,
\end{aligned}
$$

where in the last string of inequalities we used Lemma 2.5. Thus, the lemma holds for $f=\sigma$. On the other hand, from Lemma 2.4 we get that

$$
v_{2}(m !) \geq m-\frac{\log m}{\log 2}-1>\frac{3 m}{10 \log m} \quad \text { for } \quad m>50 .
$$

Thus,

$$
\begin{aligned}
v_{2}(s(m !)) & =v_{2}(\sigma(m !)-m !) \\
& \geq \min \left\{v_{2}(\sigma(m !)), v_{2}(m !)\right\} \\
& >\frac{3 m}{10 \log m} \text { for all } m>50,
\end{aligned}
$$

where we used (3.1). So, the lemma also holds for $f=s$. Finally, if we put $\ell=v_{2}(m !)$, we get $v_{2}(\phi(m !)) \geq v_{2}\left(\phi\left(2^{\ell}\right)\right)=\ell-1=v_{2}(m !)-1$. Using this and Lemma 2.4 once again, we obtain

$$
v_{2}(\phi(m !)) \geq m-\frac{\log m}{\log 2}-2>\frac{3 m}{10 \log m} \quad \text { for } \quad m>50 .
$$

Thus the lemma holds for $f=\phi$ as well. This completes the proof of the lemma.

If $2 \mid b$, then it is well known that $2 \nmid u_{n}$ for all $n \geq 1$ and so $v_{2}(f(m !))=$ $v_{2}\left(u_{n}\right)=0$, which is impossible in view of Lemma 3.1. We must therefore have that $2 \nmid b$.

Lemma 3.2. If $2 \nmid b$, then

$$
v_{2}\left(u_{n}\right) \leq v_{2}\left(u_{6} n\right)-1 \text { for all } n \geq 1 .
$$

Proof. We prove the lemma by cases according to Theorem 2.2. Since $v_{2}\left(u_{n}\right)=v_{2}(f(m !)) \geq 1$ by Lemma 3.1 , we have that the following cases are only possible: 
- If $2 \mid \Delta$ and $2 \mid n$, then $v_{2}\left(u_{n}\right)=v_{2}(n)+v_{2}(a)-1$. It also follows from Theorem 2.2 that $v_{2}\left(u_{6}\right)=v_{2}(a)$. Thus, $v_{2}\left(u_{n}\right)=v_{2}\left(u_{6} n\right)-1$.

- If $2 \nmid \Delta, \tau(2) \mid n$ and $2 \mid n$, then $v_{2}\left(u_{n}\right)=v_{2}(n)+v_{2}\left(u_{2 \tau(2)}\right)-1$. Since $2 \nmid \Delta=a^{2}+4 b$ and $b$ is odd, we get that $a$ is odd, so $\tau(2)=3$. Consequently, $v_{2}\left(u_{n}\right)=v_{2}\left(u_{6} n\right)-1$.

- Now assume that $2 \nmid \Delta, \tau(2) \mid n$ and $2 \nmid n$. So, $v_{2}\left(u_{n}\right)=v_{2}\left(u_{\tau(2)}\right)$. In this case, we have that $v_{2}(n)=0$ and $\tau(2)=3$ remains true. Additionally, by Lemma 2.3, we get $v_{2}\left(u_{3}\right) \leq v_{2}\left(u_{6}\right)-1$. These facts together imply that $v_{2}\left(u_{n}\right) \leq v_{2}\left(u_{6}\right)-1=v_{2}\left(u_{6} n\right)-1$.

Combining (1.1) and Lemmas 3.1 and 3.2 we deduce that

$$
\frac{3 m}{10 \log m}<v_{2}\left(u_{6} n\right) \text { for all } m>50 \text { and } n \geq 1 \text {, }
$$

and so $2^{\lfloor 3 m /(10 \log m)\rfloor} \mid u_{6} n$. In particular, $2^{\lfloor 3 m /(10 \log m)\rfloor} \leq\left|u_{6}\right| n$, and by applying the log function, we obtain

$$
\left\lfloor\frac{3 m}{10 \log m}\right\rfloor \leq \frac{\log \left(\left|u_{6}\right| n\right)}{\log 2} \text { for all } m>50 \text { and } n \geq 1 .
$$

On other hand, by Lemma 2.1 we get

$$
|\alpha|^{n-c \log (n+1)} \leq\left|u_{n}\right|=f(m !) \leq \sigma(m !),
$$

where $c$ is a positive constant. But

$$
\sigma(m !)=m ! \sum_{d \mid m !} \frac{1}{d}<m ! \sum_{k=1}^{m !} \frac{1}{k}<m !(1+\log m !) .
$$

Hence

$$
|\alpha|^{n-c \log (n+1)}<m !(1+\log m !) .
$$

Taking logarithms in the above inequality, we obtain that

$$
n<\frac{2(\log m !+\log (1+\log m !))}{\log |\alpha|} \text { for all } n \geq n_{0},
$$

where $n_{0}$ is a positive integer such that $2 c \log (n+1) \leq n$ for all $n \geq n_{0}$. We now apply Stirling's formula to obtain $m !<(m / 2)^{m}$, so that $\log m !<$ $m \log (m / 2)$, which itself implies that $\log (1+\log m !)<2 \log m$. Then, we deduce from (3.3) that

$$
n<\frac{2 m \log m}{\log |\alpha|} \text { for all } n \geq n_{0} .
$$

Substituting (3.4) in (3.2), we arrive at

$$
\left\lfloor\frac{3 m}{10 \log m}\right\rfloor<\frac{\log \left(2\left|u_{6}\right| m \log m / \log |\alpha|\right)}{\log 2},
$$


implying that $m \leq \max \left\{50, c_{1}\right\}$ for some effectively computable number $c_{1}$ depending only on $a$ and $b$. It then follows from (3.4) that $\max \{n, m\}$ is bounded by an effectively computable number depending only on $a$ and $b$. This completes the proof of Theorem 1.1.

\section{Some SPECIAL EXAMPLES}

In this section, we determine all the solutions of the Diophantine equation (1.1) when $\left\{u_{n}\right\}_{n \geq 0}$ is either the Fibonacci sequence or the Pell sequence. We also solve these equations for their companion sequences, namely, the Lucas and Pell-Lucas sequences, respectively. We recall that the companion sequence $\left\{v_{n}\right\}_{n \geq 0}$ of $\left\{u_{n}\right\}_{n \geq 0}$ is the sequence which satisfies the same linear recurrence relation as $\left\{u_{n}\right\}_{n \geq 0}$ but with initial values $v_{0}=2$ and $v_{1}=a$.

Better results can be obtained in these special cases for $\left\{u_{n}\right\}_{n \geq 0}$ and $\left\{v_{n}\right\}_{n \geq 0}$. Indeed, one can easily prove (by mathematical induction on $n$ ) that

$$
\alpha^{n-2} \leq u_{n} \leq v_{n} \text { for all } n \geq 1,
$$

and this provides a better lower bound for $u_{n}$ than the one given in Lemma 2.1. Here, by using (4.1) we obtain the following alternative expression for (3.4):

$$
n<\frac{m \log m}{\log \alpha}+2,
$$

which holds for all $n \geq 1$. Hence, instead of (3.5) we are led to the alternative inequality

$$
\left\lfloor\frac{3 m}{10 \log m}\right\rfloor<\frac{\log \left(u_{6} m \log m / \log \alpha+2 u_{6}\right)}{\log 2},
$$

which holds for all $m>50$.

4.1. The Fibonacci case. As mentioned before, if $(a, b)=(1,1)$, then $\left\{u_{n}\right\}_{n \geq 0}$ is the Fibonacci sequence $\left\{F_{n}\right\}_{n \geq 0}$ and so $\alpha=(1+\sqrt{5}) / 2$. In this case (4.3) becomes

$$
\left\lfloor\frac{3 m}{10 \log m}\right\rfloor<\frac{\log (8 m \log m / \log \alpha+16)}{\log 2} .
$$

This inequality implies that $m \leq 290$ and so $n \leq 3500$ in view of (4.2). A computational search of solutions of the corresponding equations in the above range allows us to state the following result.

TheOrem 4.1. We have that

- the only solutions in positive integers $(n, m)$ for the equation

$$
F_{n}=\sigma(m !)
$$

are $(1,1),(2,1),(4,2)$. 
- the only solutions in positive integers $(n, m)$ for the equation

$$
F_{n}=s(m !)
$$

are $(1,2),(2,2)$.

- the only solutions in positive integers $(n, m)$ for the equation

$$
F_{n}=\phi(m !)
$$

are $(1,1),(2,1),(1,2),(2,2),(3,3),(6,4)$.

4.2. The Pell case. With $(a, b)=(2,1)$ we obtain that $\left\{u_{n}\right\}_{n \geq 0}$ is the Pell sequence $\left\{P_{n}\right\}_{n \geq 0}$ and therefore $\alpha=1+\sqrt{2}$. Here, from (4.3) we get

$$
\left\lfloor\frac{3 m}{10 \log m}\right\rfloor<\frac{\log (70 m \log m / \log \alpha+140)}{\log 2} .
$$

In this case, we get that $m \leq 360$ and then $n \leq 2410$ by (4.2). The proof of the following theorem is completed by using Mathematica for the above range.

THEOREM 4.2. We have that

- the only solutions in positive integers $(n, m)$ for the equation

$$
P_{n}=\sigma(m !)
$$

are $(1,1),(4,3)$.

- the only solution in positive integers $(n, m)$ for the equation

$$
P_{n}=s(m !)
$$

is $(1,2)$.

- the only solutions in positive integers $(n, m)$ for the equation

$$
P_{n}=\phi(m !)
$$

are $(1,1),(1,2),(2,3)$.

4.3. The Lucas case. The Lucas sequence $\left\{L_{n}\right\}_{n \geq 0}$ is defined by $L_{0}=2$, $L_{1}=1$ and the recurrence relation $L_{n}=L_{n-1}+L_{n-2}$ for all $n \geq 2$. In 1997, Lengyel ([7]) characterized the $p$-adic valuation of the Fibonacci and Lucas numbers, as we said before. In particular, he proved that

$$
v_{2}\left(L_{n}\right)= \begin{cases}0, & \text { if } n \equiv 1,2 \quad(\bmod 3), \\ 2, & \text { if } n \equiv 3 \quad(\bmod 6), \\ 1, & \text { if } n \equiv 0 \quad(\bmod 6) .\end{cases}
$$

If $m>50$, then from Lemma 3.1 and Lengyel's result above we get that

$$
\frac{3 m}{10 \log m}<v_{2}(f(m !))=v_{2}\left(L_{n}\right) \leq 2,
$$

which is not possible. Thus, $m \leq 50$. Inserting this upper bound on $m$ into inequality (4.2) (with $\alpha=(1+\sqrt{5}) / 2$ ) we obtain that $n \leq 410$. A quick computer search in Mathematica reveals the solutions of our equations. 
THEOREM 4.3. We have that

- the only solutions in positive integers $(n, m)$ for the equation

$$
L_{n}=\sigma(m !)
$$

are $(1,1),(2,2)$.

- the only solution in positive integers $(n, m)$ for the equation

$$
L_{n}=s(m !)
$$

is $(1,2)$.

- the only solutions in positive integers $(n, m)$ for the equation

$$
L_{n}=\phi(m !)
$$

are $(1,1),(1,2)$.

4.4. The Pell-Lucas case. The Pell-Lucas sequence $\left\{Q_{n}\right\}_{n \geq 0}$ is defined by $Q_{0}=2, Q_{1}=2$ and the recurrence relation $Q_{n}=2 Q_{n-1}+Q_{n-2}$ for all $n \geq 2$. In this case, using the identity $8 P_{n}^{2}-Q_{n}^{2}=4(-1)^{n+1}$, which holds for all $n \geq 1$, and noting that $4 \mid Q_{n}^{2}$ but $8 \nmid Q_{n}^{2}$, we conclude that $v_{2}\left(Q_{n}\right)=1$ for all $n \geq 1$. From this and using Lemma 3.1 once again, we obtain that

$$
\frac{3 m}{10 \log m}<v_{2}(f(m !))=v_{2}\left(Q_{n}\right)=1,
$$

which is not possible if $m>50$. Thus, $m \leq 50$, and then $n \leq 230$ via (4.2) with $\alpha=1+\sqrt{2}$. Finally, by a computational search with Mathematica we obtain the next theorem.

THEOREM 4.4. We have that

- the Diophantine equation

$$
Q_{n}=\sigma(m !)
$$

has no solutions in positive integers $(n, m)$.

- the only solution in positive integers $(n, m)$ for the equation

$$
Q_{n}=s(m !)
$$

is $(2,3)$.

- the only solution in positive integers $(n, m)$ for the equation

$$
Q_{n}=\phi(m !)
$$

is $(1,3)$. 


\section{A general Result}

We finish this paper describing a general situation in which equation (1.1) has finitely many solutions in positive integers $n$ and $m$. In what follows, we use the Landau symbol $O$ with its usual meaning. For two sequences $\left\{a_{n}\right\}_{n \geq 0}$ and $\left\{b_{n}\right\}_{n \geq 0}$ we denote $a_{n}=O\left(b_{n}\right)$ if there exists a positive constant $K$ such that $\left|a_{n}\right| \leq K\left|b_{n}\right|$ for all sufficiently large $n$.

THEOREM 5.1. Let $\left\{u_{n}\right\}_{n \geq 0}$ be a sequence of integers such that

(i) $v_{2}\left(u_{n}\right)=O\left(n^{C}\right)$ for some constant $C \in[0,1)$, and

(ii) $n=O\left(\log \left|u_{n}\right|\right)$.

Let $f \in\{\sigma, s, \phi\}$ and consider the Diophantine equation

$$
\left|u_{n}\right|=f(m !)
$$

in positive integers $(n, m)$. Then the equation (5.1) has only a finite number of solutions.

Proof. Suppose that $(m, n)$ is an integer solution of equation (5.1) and the assumptions $(i)$ and $(i i)$ are satisfied. Note that there is only a finite number of solutions with $m \leq 50$ because $\left\{u_{n}\right\}_{n \geq 0}$ grows at least exponentially. So, we will assume that $m>50$. As in previous sections, we shall compare the 2-adic valuation on both sides of (5.1).

First, by Lemma 3.1 and the assumption $(i)$, the inequality

$$
\frac{3 m}{10 \log m}<v_{2}(f(m !))=v_{2}\left(u_{n}\right) \leq K_{1} n^{C} \quad \text { holds for all } n \geq n_{1},
$$

for some positive constant $K_{1}$ and some integer $n_{1} \geq 0$. On the other hand, by the assumption $(\mathrm{ii})$, there exist some positive constant $K_{2}$ and some integer $n_{2} \geq 0$ such that

$$
n \leq K_{2} \log \left|u_{n}\right|<K_{2}(\log m !+\log (1+\log m !)) \quad \text { for all } n \geq n_{2},
$$

which holds because $\left|u_{n}\right|=f(m !) \leq \sigma(m !)<m !(1+\log m !)$, as we have seen earlier. As in the proof of Theorem 1.1, Stirling's formula gives log $m$ ! + $\log (1+\log m !)<m \log m$, which together with (5.3) implies that

$$
n<K_{2} m \log m \text { for all } n \geq n_{2} \text {. }
$$

Combining (5.2) and (5.4), we obtain that

$$
m<K_{3}(\log m)^{K_{4}}
$$

for all $n \geq \max \left\{n_{1}, n_{2}\right\}$, where $K_{3}=\left(10 K_{1} K_{2}^{C} / 3\right)^{1 /(1-C)}$ and $K_{4}=(1+$ $C) /(1-C)$. Since $C \in[0,1)$, we have that $K_{4} \geq 1$, and so (5.5) holds only for finitely many $m$. One can in fact get an explicit upper bound on $m$ in terms of $K_{3}$ and $K_{4}$. Thus, by inequality (5.4), we obtain an upper bound for $n$. Consequently, equation (5.1) has only a finite number of solutions. Theorem 5.1 is therefore proved. 
REMARK 5.2. Let $a \in\{ \pm 2\}$ and consider the sequence $\left\{u_{n}\right\}_{n \geq 0}$ given by $u_{0}=0, u_{1}=1$ and the binary linear recurrence $u_{n}=a u_{n-1}-u_{n-2}$ for all $n \geq 2$. Here, we can easily see that $\left\{u_{n}\right\}_{n \geq 0}$ is either equal to $\{n\}_{n \geq 0}$ or to $\left\{(-1)^{n-1} n\right\}_{n \geq 0}$, and so equation (5.1) is transformed into the equation $n=$ $f(m !)$, which has infinitely many solutions in positive integers $n$ and $m$. Note that $n=O\left(u_{n}\right)$ in both cases. These examples illustrate situations in which the condition ( $\mathrm{ii}$ ) of Theorem 5.1 does not hold; however, it is usually satisfied for linear recurrence sequences. For example sequences having exponential growth. In these latter cases we only need to check whether $(i)$ holds for some constant $C \in[0,1)$.

We finish with the following corollary which describes the setting of the Fibonacci and Pell sequences for which $v_{2}\left(u_{n}\right)=O\left(v_{2}(n)\right)$.

Corollary 5.3. Let $\left\{u_{n}\right\}_{n \geq 0}$ be a sequence of integers such that

(i) $v_{2}\left(u_{n}\right)=O\left(v_{2}(n)\right)$, and

(ii) $n=O\left(\log \left|u_{n}\right|\right)$.

If $f \in\{\sigma, s, \phi\}$, then the Diophantine equation $\left|u_{n}\right|=f(m !)$ has only a finite number of solutions in positive integers $(n, m)$.

Proof. Note that $v_{2}\left(u_{n}\right)=O\left(v_{2}(n)\right)=O(\log n)=O(\sqrt{n})$. Then the result follows applying Theorem 5.1.

ACKNOWLEDGEMENTS.

We thank the reviewers for their detailed comments and suggestions which significantly contributed to improving the quality of the manuscript. The authors are members of the research group: Matemática Discreta y Aplicaciones: ERM (MATDIS).

\section{REFERENCES}

[1] A. Baker, A sharpening of the bounds for linear forms in logarithms, Acta Arith. 21 (1972), 117-129.

[2] F. Beukers, The multiplicity of binary recurrences, Compos. Math. 40 (1980), 251267.

[3] M. T. Damir, B. Faye, F. Luca and A. Tall, Members of Lucas sequences whose Euler function is a power of 2, Fibonacci Quart. 52 (2014), 3-9.

[4] L. E. Dickson, History of the Theory of Numbers. Vol. I: Divisibility and Primality, Chelsea Publishing Co., New York, 1966.

[5] D. Iannucci and F. Luca, Catalan numbers, factorials, and sums of aliquot parts, Fibonacci Quart. 45 (2007), 327-336.

[6] K. K. Kubota, On a conjecture of Morgan Ward I, II, Acta Arith. 33 (1977), 11-28, 29-48.

[7] T. Lengyel, The order of the Fibonacci and Lucas numbers, Fibonacci Quart. 33 (1997), 234-239.

[8] F. Luca, Arithmetic functions of Fibonacci numbers, Fibonacci Quart. 37 (1999), $265-268$.

[9] F. Luca and M. Mignotte, $\phi\left(F_{11}\right)=88$, Divulg. Mat. 14 (2006), 101-106. 
[10] F. Luca and F. Nicolae, $\phi\left(F_{m}\right)=F_{n}$, Integers 9 (2009), A30.

[11] F. Luca and P. Pollack, Multiperfect numbers with identical digits, J. Number Theory 131 (2011), 260-284.

[12] F. Luca and P. Stanica, Equations with arithmetic functions of Pell numbers, Bull. Math. Soc. Sci. Math. Roumanie 57(105) (2014), 409-413.

[13] P. Lucas, Théorie des fonctions numériques simplement périodiques, Amer. J. Math. 1 (1878), 184-240.

[14] D. Marques, The order of appearance of product of consecutive Fibonacci numbers, Fibonacci Quart. 50 (2012), 132-139.

[15] M. Renault, The period, rank, and order of the $(a, b)$-Fibonacci sequence Mod $m$, Math. Mag. 86 (2013), 372-380.

[16] P. Ribenboim, My numbers, my friends: popular lectures on number theory, SpringerVerlag, New York, 2000.

[17] J. B. Rosser and L. Schoenfeld, Approximate formulas for some functions of prime numbers, Illinois J. Math. 1 (1962), 64-94.

[18] C. Sanna, The p-adic valuation of Lucas sequences, Fibonacci Quart. 54 (2016), $118-124$.

[19] A. Schinzel, Primitive divisors of the expression $A^{n}-B^{n}$ in algebraic number fields, J. Reine Angew. Math. 268(269) (1974), 27-33.

[20] A. Schinzel, Primitive divisors of Lucas and Lehmer numbers, in: Transcendence Theory: Advances and Applications, Academic Press Inc, London, 1977, 79-92.

[21] N. J. Sloane et al., The on-line encyclopedia of integer sequences, published electronically at https://oeis.org, 2021.

E. F. Bravo

Departamento de Matemáticas

Universidad del Cauca

Calle 5 No. 4-70 Popayán

Colombia

E-mail: fbravo@unicauca.edu.co

J. J. Bravo

Departamento de Matemáticas

Universidad del Cauca

Calle 5 No. 4-70 Popayán

Colombia

E-mail: jbravo@unicauca.edu.co

Received: 16.7.2020.

Revised: 8.9.2020. 